Methods Mortality caused by external causes provided by the Vital Statistics of Japan and the standard population data were used.

Results Among 0-14 year olds, mortality due to external causes declined from 13.1 per 100,000 population in 1984 to 3.6 in 2014. Of the types of external causes, suicide death rate increased, but drowning, motor vehicle crashes and suffocation death rate were reduced.

Among 15-14 year olds, mortality due to external causes declined from 36.8 per 100,000 population in 1984 to 26.2 in 2014. Of the types of external causes, suicide death rate increased, but drowning and motor vehicle crashes death rate were reduced.

Among 45-64 year olds, mortality due to external causes declined from 63.6 per 100,000 population in 1984 to 42.1 in 2014. Of the types of external causes, suffocation death rate increased, but motor vehicle crashes and suicide death rate were reduced.

Among those 65 years or older, mortality due to external causes declined from 135.2 per 100,000 population in 1984 to 105.1 in 2014 . Of the types of external causes, suffocation and drowning death rate increased, but suicide, motor vehicle crashes and fall death rate were reduced.

Conclusions In Japan, the motor vehicle crashes death rate among all ages decreased significantly. The drowning and suffocation death rate among children decreased, but among those 65 years or older those rates increased.

Laws requiring seat belts from 1985 and child seat under 6 year old from 2000 have prevented many traffic fatalities.

Development of such legal systems would be useful for a decline of the death rate mortality due to external causes.

\section{UNIQUE FEATURES OF MORTALITY, HOSPITALIZATIONS AND OUT-PATIENT VISITS DUE TO EXTERNAL CAUSES BY AGE GROUP IN JAPAN}

${ }^{1}$ Yoshiaki Ikemi, ${ }^{2}$ Tetsuro Tanaka, ${ }^{2}$ Michio Tanaka, ${ }^{3}$ Yuko Uchiyama, ${ }^{4}$ Hiroko Ishii. ${ }^{1}$ Kanto Gakuin University Graduate School of Engineering, Japan; ${ }^{2} J a p a n$ Child Policy Research and Promotion, Japan; ${ }^{3} J a p a n$ Women's College of Physical Education, Japan; ${ }^{4}$ Second Naminori Nursery, Japan

\subsection{6/injuryprev-2016-042156.953}

Background The purpose of this study is to analyse the unique features of mortality, hospitalizations and out-patient visits due to external causes in Japan and to explore strategies for injury prevention.

Methods The WHO standard population data and data on mortality caused by external causes made available by the Vital Statistics of Japan were used. Also, the rates of hospitalizations and outpatient visits due to unintentional injuries were calculated using the Patient Survey of Japan.

The deaths caused by the Great East Japan Earthquake in 2011 were removed from the analysis to avoid confounding.

Results Among all ages, the mortality due to external causes was 59.7 per 100,000 populations in 2011. Among 0-14 year olds, the mortality due to external was 3.9 per 100,000 populations. Among 15-44 year olds, the mortality due to external causes was 31.1 per 100,000 populations. Among 45-64 year olds, the mortality due to external causes was 51.1 per 100,000 populations. Among those 65 years or older, the mortality due to external causes was 143.9 per 100,000 populations.
Among all ages, assuming injury death was one, the hospitalizations were 18 and out-patient visits were 286. Among 0-14 year olds, assuming injury death was one, the hospitalizations were 123 and out-patient visits were 9,548. Among 15-44 year olds, assuming injury death was one, the hospitalizations were 20 and out-patient visits were 508. Among 45-64 year olds, assuming injury death was one, the hospitalizations were 15 and outpatient visits were 242. Among those 65 years or older, assuming injury death was one, the hospitalizations were 18 and outpatient visits were 110 .

Conclusions Among 0-14 olds, the mortality due to external causes and rate of hospitalizations were lower than another age groups, but rate of out-patient visit was higher.

Among 15-44 year olds and among 45-64 year olds, rate of hospitalisation was higher than children, but rate of out-patient visit was half of them.

Among those 65 years or older, the mortality due to external causes and rate of hospitalizations were higher than another age groups, but rate of out-patient visit was half of children.

\section{ANIMAL INJURY MORTALITIES AND MORBIDITIES: EVIDENCE FROM A COMMUNITY SURVEY}

'Al-Amin Bhuiyan, ${ }^{1}$ Kamran UI Baset, ${ }^{2}$ Olakunle Alonge, ${ }^{1}$ Aminur Rahman, ${ }^{1}$ Fazlur Rahman, ${ }^{2}$ Adnan A Hyder. ${ }^{1}$ Centre for Injury Prevention, Health Development and Research, Bangladesh (CIPRB); ${ }^{2}$ Johns Hopkins University, USA

\subsection{6/injuryprev-2016-042156.954}

Background Animal injuries are the major causes of morbidity and mortality worldwide including developing countries. Worldwide, up to five million people are bitten by snakes every year; the majority in Africa and South-East Asia. Dog bites account for tens of millions of injuries annually; the highest risk is among children. In Bangladesh, the most of the animal injuries including snake and dog bites are common. This study determined the magnitude and pattern of animal injury mortalities and morbidities in rural Bangladesh.

Methods A cross sectional survey was conducted in 19 Unions of 3 sub-districts of Bangladesh from June to September 2013. Socio-economic, demographic, environmental and injury related data were collected from adult respondents by face-to-face interview with the help of structured questionnaires at a household level. A total of 534,221 population of all ages were studied from 128,820 households.

Results The incidence of fatal and non-fatal animal injury in all ages were 1.2 (95\% CI: 0.4-3.4) and 1,165.4 (95\% CI: 1128.8 $1203.1)$ per 100,000 population respectively. The highest nonfatal animal injury rate 1,367.0 (95\% CI: 1310.7-1425.7) per 100,000 population was observed in the age group 25-64 years. The morbidity was found higher among males across all ages. $65.0 \%$ of the animal injuries occurred without any provocation. The highest proportion $(28.2 \%)$ of animal injuries was caused by the cattle, which was followed by wasp $24.4 \%$, dog $11.3 \%$, hornet $9.6 \%$, and snake $6.4 \%$ bites.

Conclusions Above data support that animal injury is an important public health issue in Bangladesh. Immediate attention should be made to strengthen the intervention measures to prevent these unexpected events. 